\title{
COMPLIANT PARALLEL ROBOTS
}

\author{
Development and Performance
}

Annika Raatz, Jan Wrege, Arne Burisch and Jürgen Hesselbach

Institute of Machine Tools and Production Technology (IWF), Technical University Braunschweig, Langer Kamp 19b, 38106 Braunschweig

\begin{abstract}
In this paper the development of a macro parallel robot is presented in which conventional bearings are replaced by pseudo-elastic flexure hinges. The robot consists of a spatial parallel structure with three translational degrees of freedom and is driven by three linear direct drives. The structure has been optimized with respect to workspace and transmission ratio. Additionally, in simulations with the FEA tool ANSYS different geometrical arrangements and combinations of flexure hinges have been investigated with respect to the dynamic behavior of the compliant mechanism. Due to the symmetrical character of the structure and the optimized design of the combined flexure hinges the structure is very stiff. The experimental measured repeatability of the compliant robot is below $0.3 \mu \mathrm{m}$.
\end{abstract}

Key words: High Precision Robotics, Compliant Mechanism, pseudo-elastic SMA, flexure hinges

\section{INTRODUCTION}

The suitability of robots, which can be used for micro assembly tasks, depends on the performance specifications referred to accuracy. Usually different terms are used to describe these specifications. Slocum ${ }^{1}$ explains these terms in a very demonstrative way: Accuracy is the ability to tell the truth, repeatability is the ability to tell the same story each time and resolution is the detail to which you tell a story. A more formal definition for these terms is given in the ISO standard EN ISO 9283 and specifies appropriate test methods. The accuracy of a robot is generally influenced by systematic and stochastic failures. While systematic failures can be compensated directly by the robot control, if they are known, stochastic 
failures can not. These can be caused by the travel of bearing cage and clearance in the joints of a robot. Due to their structure, parallel robots are typically built up with a high number of joints. These joints mostly have more than one degree of freedom which may result in a decreasing precision. For high precision assembly tasks in microsystem technology, these problems have been overcome by using flexure hinges instead of conventional rotational joints. ${ }^{2,3,4}$ The integration of flexure hinges in parallel structures is relatively simple because, except for the drives, all joints are passive. Since flexure hinges gain their mobility exclusively from a deformation of matter, they do not posses the above named disadvantages of conventional joints. The allowable strains of the deformed hinge material are the limiting factor for the attainable angle of rotation. Basic parameters accounting for the maximal angle of rotation are the geometry, geometrical dimensions and the elastic and plastic material properties. Designing flexure hinges is always a compromise between accuracy, compact over-all dimensions, high mobility, out of axis stiffness and the aspired life cycle of the product. At present flexure hinges are mainly used in small devices like micro-positioning devices and micro-grippers with small angular deflections and limited workspaces, but high accuracies. ${ }^{5,6,7}$

In this paper an approach for using flexure hinges in a macro parallel robot, which offers a larger workspace than typical compliant mechanism is presented. Conventional joints were replaced with flexure hinges made of shape memory alloy. Due to large reversible strains of the used material, maximal angular deflections of the used hinges of $\pm 30^{\circ}$ are possible. This high mobility can be achieved with high kinematic accuracy and a relative small design space at the same time. This offers the possibility to design robots with high accuracy and resolution with sufficiently large workspaces for typical micro assembly tasks.

\section{FLEXURE HINGES}

A wide variety of different designs of flexure hinges are proposed in the literature. ${ }^{8,9,10}$ They are designed monolithic or hybrid allowing for up to 3 degrees of freedom (DOF). In most cases it is tried to achieve high angular deflections with small occurring elastic strains. Using pseudo-elastic SMA for flexure hinges offers the possibility to design hinges with large angular deflections and small kinematic deviations at the same time. For the flexure hinges in this robot a pseudo-elastic CuAINiFe single crystal SMA is used. This SMA has a superior machinability than NiTi-SMA and offers reversible strains up to $17 \%$. Fig. 1(a) shows the well known flexure beam and flexure notch hinge. Generally, the notch hinge permits smaller angular deflections 
than the flexure beam hinge but it has an advanced kinematic behavior and is more insusceptible against unintentional deformations and buckling.

Kinematic deviations are caused by the instantaneous centre of rotation which has no fixed position relative to the rigid links but moves during deflection. ${ }^{11,12}$ The deviation $\Delta \mathrm{r}_{\mathrm{fh}}$ is calculated by the difference of the coordinates of point $\mathrm{E}$ (Fig. 1) lying on the deflection curve of the hinges and the ideal joint. The deviation depends mainly on geometry, geometrical dimensions and deflection of the hinge. The larger the part of the hinge where the deformation takes place the larger the deviation ${ }^{13}$. For the compliant robot, pseudo-elastic flexure notch hinges with $\mathrm{R}=15 \mathrm{~mm}$ and $\mathrm{h}=0.15 \mathrm{~mm}$ are used $(1=27 \mathrm{~mm})$. These geometrical dimensions are an optimum between small kinematic deviations and small occurring strain rates. The resulting deviations are $67 \mu \mathrm{m}$ at $30^{\circ}$ deflection. This flexure notch hinge is comparable to a flexure beam hinge with $l_{h}=2 \mathrm{~mm}$, though easier to manufacture.

The movement of the instantaneous centre of rotation is also reflected in the kinematic behavior of the robot with integrated flexure hinges (compliant mechanism). The compliant mechanism was simulated by means of FEA. For certain positions of the tool centre point (TCP) the displacements of the drives have been computed solving the inverse kinematic problem (IKP) of the rigid body model. These displacement values where used as constraints for the nodes representing the drives in the FE-model. The difference $\Delta \mathrm{r}_{\mathrm{CM}}$ between the analytically computed TCP and the TCP of the compliant model can be determined within the whole workspace with Eq. (1).

$$
\Delta r_{C M}=\sqrt{\left(x_{T C P, R B M}-x_{T C P, C M}\right)^{2}+\left(y_{T C P, R B M}-y_{T C P, C M}\right)^{2}+\left(z_{T C P, R B M}-z_{T C P, C M}\right)^{2}}
$$

Starting from the undeformed initial position of the compliant mechanism the deviations are increasing with increasing displacement of the TCP (Fig. 2). Reasons are the larger deflections of the hinges and the accompanying increasing deviations compared to ideal joints.

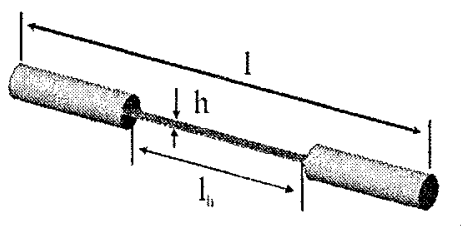

a)

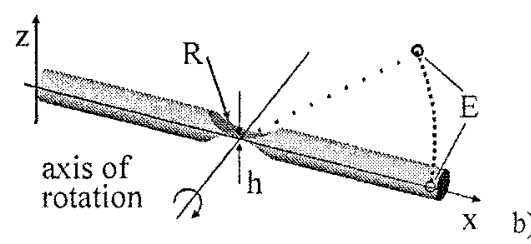

Figure 1. Flexure beam (a) and notch (b) hinge with 1 DOF 


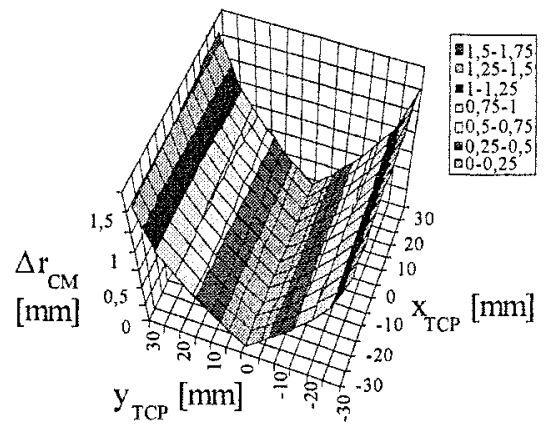

Figure 2. Kinematic deviation of the compliant mechanism (CM) and the rigid body model (RBM) with ideal rotational joints $\left(\mathrm{z}_{\mathrm{TCP}}=\right.$ constant $)$

For example a planar movement in an area of $60 \times 60 \mathrm{~mm}^{2}$ leads to maximal deviation of about $1.5 \mathrm{~mm}$ and if the movement is $60 \mathrm{~mm}$ in $z$ direction the deviation is only about $0.05 \mathrm{~mm}$. The simulations indicate that flexure hinges principally reduce the absolute positioning accuracy. The error is induced when describing the compliant mechanism by means of a rigid body model, neglecting the different kinematic behavior of flexures hinges compared to ideal joints. In consideration of the high number of flexure hinges the TCP deviation is relatively small but not categorically negligible. There exist approaches to increase the absolute accuracy of a compliant robot, e.g. by adding additional actuators ${ }^{7}$ or by varying the link lengths in the model depending on the deflection of the hinges ${ }^{14}$. To succeed with these approaches a very accurate calibration of the robot is required. Depending on the application it has to be decided whether the effort to compensate for the deviation is necessary. For many assembly tasks a high resolution and repeatability should be sufficient ${ }^{15}$, which are not influenced by these kinematic deviations.

\section{STRUCTURE OF THE PARALLEL ROBOT}

In the following section a short description of the structure of the chosen parallel robot is given (Fig. 3) ${ }^{16,17}$ The structure is a variant of the "Delta" robot of Clavel ${ }^{18}$ and the "Star" robot of Hervé ${ }^{19}$. The robot consists of a spatial parallel structure with three translational degrees of freedom and is driven by three linear drives. The platform is connected with each drive by two links forming a parallelogram and allowing only translational movements of the platform and keeping the platform parallel to the base plane. The three drives of the structure are arranged in the base plane at intervals of $120^{\circ}$ star-shaped. Thus the structure has a workspace which is 
nearly round or triangle-shaped. By restricting the angular deflection of the flexure hinges the workspace is reduced and also changes slightly its shape. With a design angle of $\alpha_{0}=34^{\circ}$ the workspace of the structure is optimized with respect to dimension and shape (Fig. 4). The angle $\alpha_{0}$ is the angle between the planes of the parallelograms and the working platform in the initial position of the structure with no deflections of the hinges (Fig. 3).

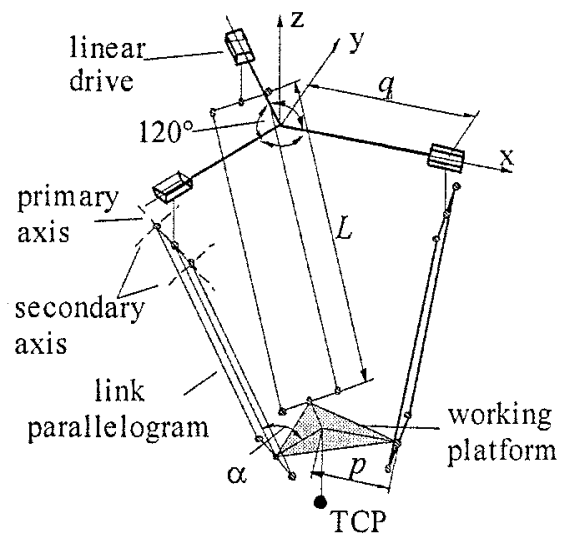

Figure 3. Structure of the parallel robot (Triglide ${ }^{5}$ )

a)

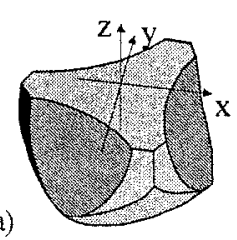

b)

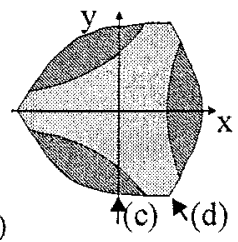

c)

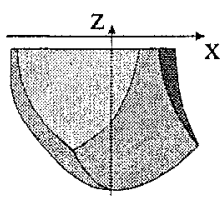

d)

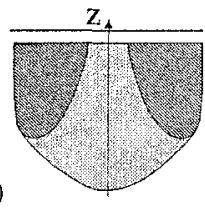

Figure 4. Optimized workspace of the compliant robot: a) spatial view, b) top view

Table 1 gives the results of this workspace optimization. The displacement of the drives is restricted to $\Delta q=315 \mathrm{~mm}$ and the geometrical dimensions are chosen to $\mathrm{L}=350 \mathrm{~mm}$ and $\mathrm{p}=45 \mathrm{~mm}$. The restriction of the hinges deflections of $\pm 30^{\circ}$ reduces the workspace only minimal compared to the workspace with no angular restriction. The workspace reaches its maximum in the plane with $\mathrm{z}=-195 \mathrm{~mm}$. The shape is roughly a circle with a diameter of $300 \mathrm{~mm}$. Using flexure hinges with conventional spring steel (maximal elastic strains of $0.5 \%$ ) the angular deflection is restricted to $\pm 5^{\circ}$. In this case the resulting workspace of the structure is hundred times smaller compared to the workspace using pseudo-elastic flexure hinges. A restriction of the hinges deflection to $\pm 20^{\circ}$ decreases the workspace by the factor two. 
Table 1. Workspace dimensions of the structure

\begin{tabular}{lllll}
\hline Deflection & $x \min , \max [\mathrm{mm}]$ & $y \min , \max [\mathrm{mm}]$ & $\begin{array}{l}\mathrm{z} \min , \max \\
{[\mathrm{mm}]}\end{array}$ & workspace \\
\hline $\pm 180^{\circ}$ & $-218,176$ & $-206,206$ & $-324,0$ & 1 \\
$\pm 30^{\circ}$ & $-202,176$ & $-174,174$ & $-314,-26$ & 0.88 \\
$\pm 20^{\circ}$ & $-138,138$ & $-118,118$ & $-282,-84$ & 0.45 \\
$\pm 5^{\circ}$ & $-34,34$ & $-30,30$ & $-220,-170$ & 0.008 \\
\hline
\end{tabular}

Due to the symmetric structure and the chosen length of the legs a homogeneous transmission behavior from the drives to the working platform is obtained. The dimensions of the structure are calculated in a way that the transmission ratio $t$ from platform to drive is smaller than 1 within the used workspace $(190 \mathrm{~mm}<\mathrm{z}<250 \mathrm{~mm})$. Theoretically this enables a more accurate positioning of the platform than the drives themselves. ${ }^{17,20}$

\section{DESIGN OF COMBINED FLEXURE HINGES}

One problem of flexure hinges and compliant mechanism is that the hinges act as springs with restoring forces. Without any damping component, except for the inner material damping, the compliant mechanism normally tends to vibrate due to the compliance. In various FE-simulations, different geometrical arrangements and combinations of flexure hinges have been investigated with respect to the dynamic behavior (natural frequencies and forms) of the compliant mechanism.

The spatial parallelogram of the structure is built with one primary axis, connected to the platform and the drives, respectively, and two secondary axes, at which the parallelogram rods are connected. The primary and the secondary axes must intersect to allow for two DOF. The flexure hinges with one DOF (Fig. 1) has to be combined in such a way offering two DOF with intersecting rotational axes (Fig. 5). This can be done either by arranging the flexure hinges of the primary axis between the flexure hinges of the secondary axes or on the outside of the secondary axes.
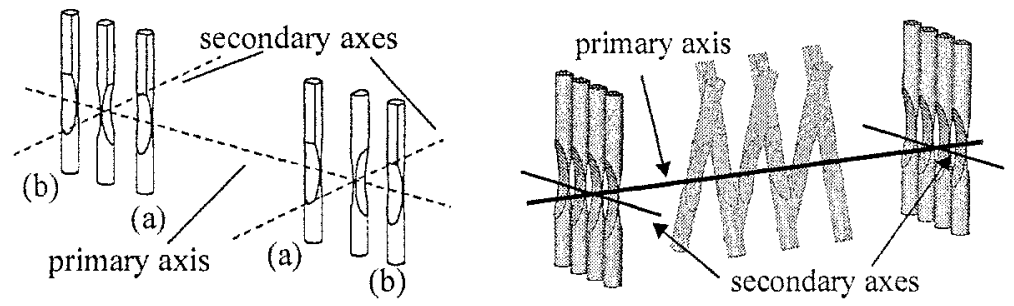

Figure 5. Rotational axes of the flexure hinges and axes of the spatial parallelogram of the structure (left); final design of combined flexure hinges (right) 
Fig. 5(left) (a) shows the design variant with the primary axis inside of the secondary axes (i-pa design) and Fig. 5(left) (b) the design variant with the primary axis outside of the secondary axes (o-pa design). With additional spectrum analyses and transient analyses the frequencies of the structures could be determined which have the largest influence. Though the o-pa design seems to be a bit stiffer, the design variant with inner primary axis was chosen for the final robot design due to the possibility of a more compact design. Fig. 5(right) shows the combination of single flexure hinges. Due to parallel and angular arrangement of the hinges, the torsional moments can be better absorbed and transformed into tension and compression forces.

\section{COMPLIANT PARALLEL ROBOT}

The final design of the compliant robot consists of 84 discrete flexure hinges and is built with CFK rods to minimize the moved mass which is $1120 \mathrm{~g}$ (Fig. 6). The robot is driven by linear direct drives with an incremental measuring system with a resolution of 0.125 micrometer. An additional rotational axis can be mounted on the working platform to adjust the orientation of the end-effector. The footprint of the robot is $\sim 1280 \times 980$ $\mathrm{mm}^{2}$ and with the actual configuration, a cube with a dimension of $112 \times 112$ $\mathrm{x} 112 \mathrm{~mm}^{3}$ fits into the workspace. Due to symmetrical arrangement of the structure with respect to the support of the working platform and optimized combinations of flexure hinges a quite rigid structure was built and occurring vibrations are damped quite fast. Static FE-simulations of the robot in its initial position leads to a stiffness coefficient of $530 \mathrm{~N} / \mathrm{mm}$ in $\mathrm{z}$-direction, which is the main joining direction. In the $x y$-plane the stiffness coefficient is smaller $(\sim 200 \mathrm{~N} / \mathrm{mm})$, but also more than sufficiently for micro assembly tasks.

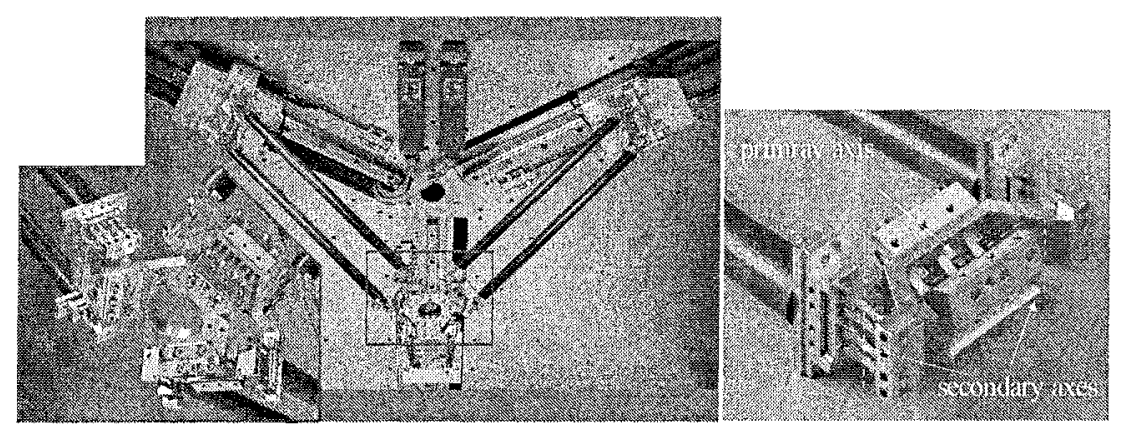

Figure 6. Compliant robot with $3 \mathrm{DOF}$ and optimized design of a flexure hinge with $2 \mathrm{DOF}$ 
A modal analysis of the final design leads to natural frequency of $f_{1}=68.7 \mathrm{~Hz}, f_{2}=70.74 \mathrm{~Hz}$ und $f_{3}=120.7 \mathrm{~Hz}$. The simulations were experimentally validated with deviations less than $8 \%\left(f_{1, e x}=65 \mathrm{~Hz}\right.$, $f_{2, e x}=68 \mathrm{~Hz}$ und $f_{3, e x}=112 \mathrm{~Hz}$ ). The measurement of the second and third natural frequency is shown in Fig. 7. The first frequency could not be measured simultaneously. With measurements against the frame of the robot the first peaks with frequencies below $20 \mathrm{~Hz}$ could be identified as vibrations of the frame and basement. These are no vibrations of the structure itself.
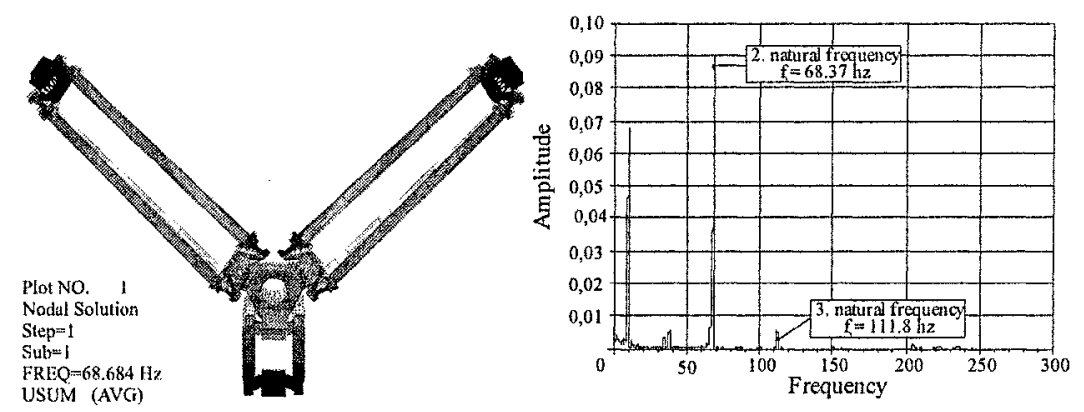

Figure 7. FEM simulation of the compliant mechanism and experimental validation: Plot of the first mode with an natural frequency of $68 \mathrm{~Hz}$ (left) and measured natural frequencies

The performance of the robot has been investigated by measuring the repeatability and the variance of multiple direction position accuracy. Capacitive sensors with a resolution of $8 \mathrm{~nm}$ have been used and the measures and calculations have been carried out according to EN ISO 9283. The repeatability (RP) is computed according to equation 3 where 1 denotes the mean deviation from the centroid of subsequent actual poses and $S_{I}$ denotes the standard deviation. Graphically the repeatability can be described as the radius of a sphere in which $99.7 \%$ of all subsequent poses lie (Fig. 8).

$$
R P=\bar{l}+3 S_{l}
$$

The variance of the multiple direction position accuracy (vAP) expresses the deviation between the different average actual poses which result from approaching the same desired pose from three directions orthogonal to each other. This value is important for micro assembly tasks in which the endeffector has to move to the same position from different directions. Especially at this kind of robot movements, backlash in conventional joints plays a major role and this value vAP is often much worse than the 
repeatability $R P .^{21}$ The experimental investigations with the compliant robot lead to a repeatability below $R P=0.3 \mu \mathrm{m}$ (best values were $R P_{x y z}=0.19 \mu \mathrm{m}$ and in the $x y$-plane $\mathrm{RP}_{\mathrm{xy}}=0.13 \mu \mathrm{m}$ ) and a variance of multiple direction position accuracy of $v A P=0.15 \mu \mathrm{m}$. These are very good results and could be even improved by increasing the resolution of the linear encoders.

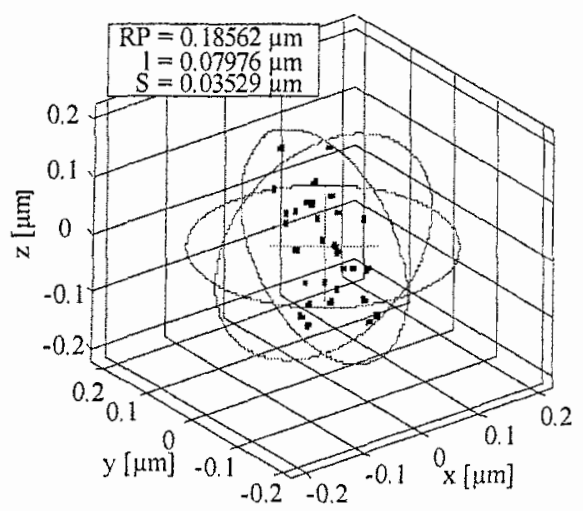

Figure 8. Measured repeatability of the robot

\section{SUMMARY}

This paper presents the design of a spatial compliant parallel robot with pseudo-elastic flexure hinges. The robot has three degrees of freedom and a workspace larger than $200 \times 200 \times 60 \mathrm{~mm}^{3}$. The compliant mechanism and the design of the flexure hinges have been optimized with respect to workspace dimension, transmission ratio and stiffness. The large workspace could be achieved due to the pseudo-elastic material of the flexure hinges. The lack of backlash, friction and stick-slip effects within the mechanism lead to a repeatability below $0.3 \mu \mathrm{m}$ and a resolution better than $0.125 \mu \mathrm{m}$, respectively. 


\section{REFERENCES}

1. A. H. Slocum, Precision Engineering Research Group (07/2004); http://pergatory.mit.edu.

2. S. L. Canfield, J. W. Beard, N. Lobontiu, E. O'Malley, M. Samuelson, and J. Paine, Development of a spatial compliant manipulator, International Journal of Robotics and Automation 17(1), 63-71 (2002).

3. R. Clavel, P. Helmer, T. Niaritsiry, S. Rossopoulos, and 1. Verettas, High precision parallel robots for Micro-Factory applications, Robotic Systems for Handling and Assembly, 285-296 (2005).

4. W. Dong, Z. Du, and L. Sun, Conceptional design and kinematics modeling of a widerange flexure hinge-based parallel manipulator, Proc. of the 2005 IEEE Int. Conference on Robotics and Automation, $4042-4047$ (2005).

5. M. L. Culpepper, and G. Anderson, Design of a low-cost nano-manipulator which utilizes a monolithic, spatial compliant mechanism, Precision engineering 28, 469-482 (2004).

6. M. Goldfarb, and N. Celanovic, A flexure-based gripper for small-scale manipulation, Robotica, 17(2), 181-187 (1999).

7. B.-J. Yi, H.-Y. Na, G. B. Chung, W. K. Kim, and I. H. Suh, Design and experiment of a 3 dof parallel micro-mechanism utilizing flexure hinges, Proc. of ICRA 19(4), 1167-1172 (2003).

8. S.T. Smith, Flexures: Elements of Elastic Mechanisms (Gordon \& Breach Science Publishers, 2000).

9. N. Lobontiu, Compliant Mechanisms - Design of Flexure Hinges (CRC Press, 2003).

10. B. D. Jensen, and L. L. Howell, The modeling of cross-axis flexural pivots, Mechanism and machine theory 37, 461-476 (2001).

11. L. L. Howell, and A. Midha, Parametric deflection approximations for end-loaded, largedeflection beams in compliant mechanisms, Journal of Mechanical Design 117(3), 156165 (1995).

12. J. Hesselbach, A. Raatz, Pseudo-elastic flexure-hinges in robots for micro assembly, Proc. of SPIE Microrobotics and Microassembly II, 4194, 157-167 (2000).

$13 \mathrm{~J}$. Hesselbach, A. Raatz, and H. Kunzmann, Performance of pseudo-elastic flexure hinges in parallel robots for micro-assembly tasks, Annals of the CIRP 53(1), 239-332, (2004).

14. R. Thoben, Parallelroboter für die automatisierte Mikromontage (Fortschritt-Berichte VDI Verlag, 1999).

15. P. Schellekens, N. Rosielle, H. Vermeulen, S. Wetzels, and W. Pril, Design for precision: Current status and trends, Annals of the CIRP 47(2), 557-586 (1998).

16. J. Hesselbach, J. Wrege, O. Becker, and S. Dittrich, A micro-assembly-station based on a hybrid 4-dof-robot, Proc. of the Int. Precision Assembly Seminar, 55-61 (2003).

17. J. Hesselbach, O. Becker, S. Dittrich, and P. Schlaich, A new hybrid 4-d.o.f.-robot for micro-assembly, Production Engineering IX(1), 105-108 (2002).

18. R. Clavel, DELTA, a fast robot with parallel geometry, Proc. of 18 th Int. Symposium on Industrial Robot, 91-100 (1988).

19. J. M. Hervé, and F. Sparacino, Star, a new concept in robotics, Proc. of the $3 \mathrm{rd}$ Int. Workshop on ARK, 176-183 (1992).

20. A. Raatz, J. Wrege, N. Plitea, and J. Hesselbach, High precision compliant parallel robot, Production Engineering XII(1), 197-202 (2005).

21. J. Hesselbach, G. Pokar, J. Wrege, and K. Heuer, Some aspects on the assembly of active micro systems, Production Engineering XI(1), 159-164 (2004). 